\title{
A Rare Case of Vanishing Bone Disease of Metacarpals
}

\author{
Prashant Kamble ${ }^{1}$, Ashwin Sathe ${ }^{1}$, Shubhranshu Mohanty ${ }^{1}$, Tushar Rathod ${ }^{1}$
}

\author{
Learning Point of the Article:
}

The diagnosis of the vanishing bone disease should be made with clinical, radiological, and histopathological evaluation.

\section{Abstract}

Introduction: Vanishing bone disease is rare phenomenon of idiopathic origin that leads to extensive osteolysis of bone. Prognosis of disease is unpredictable and definitive guidelines for management are still unknown. The vanishing bone disease has been reported for multiple other bones, however, this probably is the 1st time that vanishing bone disease of the metacarpals is being reported.

Case Report: A 22-year-old male presented with shortening middle finger and poor grip strength of the left hand. Serial radiographs revealed progressive concentric reduction of third and then fourth metacarpal shaft, with a sucked candy appearance. All the blood parameters were normal including calcium and parathormone levels. There was no evidence of any tumor elsewhere in the body. Biopsy showed myxoid areas, proliferating vessels interposed with skeletal muscles. Thus, based on clinical, radiological, and histopathological findings, we made the diagnosis of vanishing bone disease. The patient was treated with autologous non-vascularized fibula graft and was fixed with transverse k-wires to adjacent metacarpals. At 2-year follow-up, graft was completely incorporated and the patient gained full functional recovery.

Conclusion: Vanishing bone disease affecting the metacarpals is very rarely reported in the literature. The diagnosis should be made by excluding all the other conditions such as primary bone tumors or secondary from other sites. There are no fixed treatment guidelines. However, we could treat this condition successfully with autologous non-vascularized fibular graft.

Keywords: Vanishing bone disease, osteolysis, metacarpal, hand surgery.

\section{Introduction}

Vanishing bone disease is rare phenomenon of unknown origin that leads to extensive osteolysis of bone. Vanishing bone disease is otherwise known as Gorham's disease or idiopathic osteolysis of uncertain etiology. The first description of vanishing bone disease was given by Jackson in humerus. In 1955 , Gorham and stout played a key role in publishing an article describing massive osteolysis in a disease with hemangiomatosis [1]. Vanishing bone disease is very rare with no age, gender, or race predilection [2]. There are several theories proposed for etiology of vanishing bone disease. At the cellular level, the disease is thought to occur because of increased osteoclastic activity along with proliferating endothelial lined lymphatic vessels [3]. The usual clinical features are gradual onset of pain, functional disability, and deformity of affected part secondary to pathological fractures. Vanishing bone disease can affect one or multiple bones with either spontaneously resolving behavior or progressively worsening nature. There are a few cases reported in the literature, affecting upper extremities, maxillofacial region, some involving pelvis and cervical spine [4]. Some fatal complications such as extensive lymphangiectasia in pleura or thoracic duct have also been reported, when disease affects ribs and thoracic cage [5]. In this case report, we present a case of vanishing bone disease of metacarpals which we successfully treated with non-vascularized fibula grafting.

\section{Case Report}

A 22-year-old right hand dominant male presented in the outpatient department with complaints of shortening and inability to extend middle finger of the left hand. On examination, there was an extension lag at the middle finger, poor grip strength, and reduced height of middle ray (Fig. 1). The patient had a history of incidental trauma to the left hand for which he was treated conservatively. Over the 6 months after the trauma, the patient started developing the symptoms in the form of gradual shortening and reduced handgrip strength and presented to our hospital. On investigation, blood parameters including calcium levels and parathormone levels were normal. The X-ray showed pathological fracture of third metacarpal with osteolytic lesion involving base of second metacarpal and carpal bones (Fig. 2).

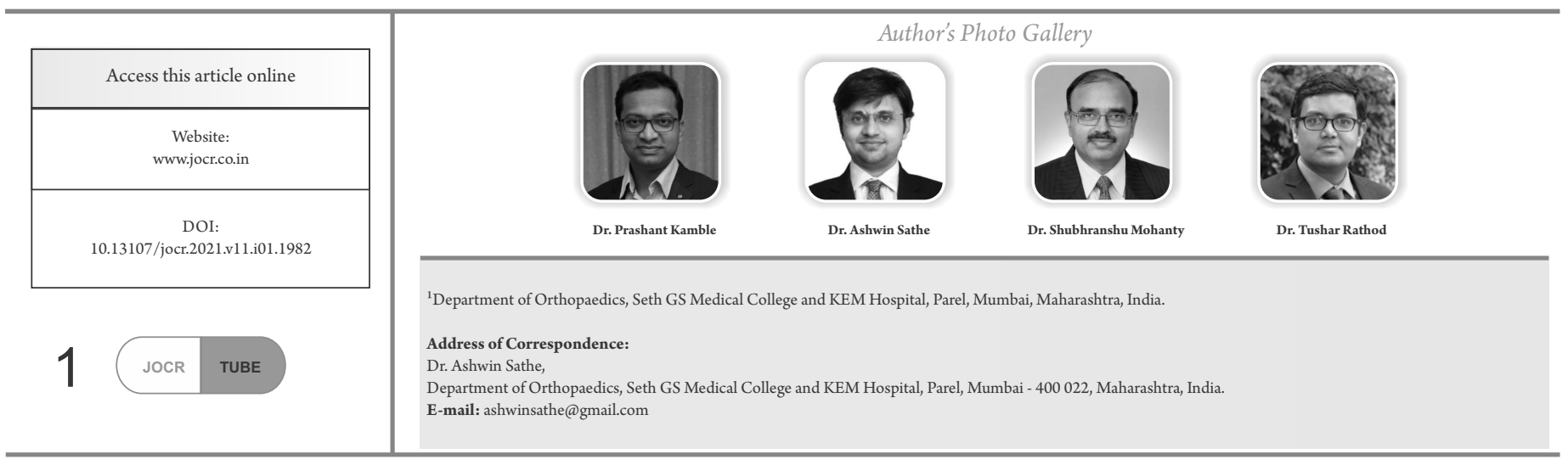

Journal of Orthopaedic Case Reports | pISSN 2250-0685 | eISSN 2321-3817 | Available on www.jocr.co.in | doi:10.13107/jocr.2021.v11.i01.1982 This is an Open Access article distributed under the terms of the Creative Commons Attribution Non-Commercial License (http://creativecommons.org/licenses/by-nc/3.0) which permits unrestricted non-commercial use, distribution, and reproduction in any medium, provided the original work is properly cited. 


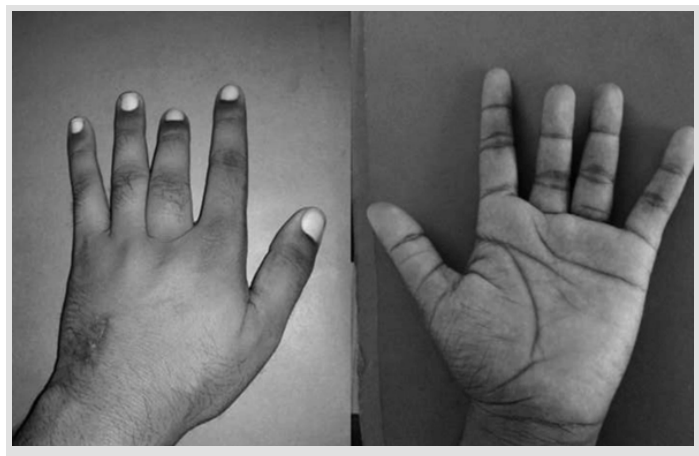

Figure 1: Clinical photograph of the patient at presentation.

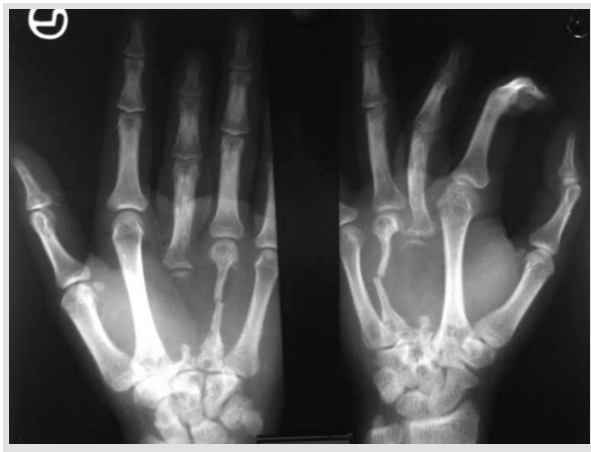

Figure 2: Radiograph of the affected hand at the time of initia presentation.
Magnetic resonance imaging showed almost complete osteolysis of the 3rd metacarpal with only the base visible and fracture of the 4th metacarpal with thinning and resorption of the bone and surrounding soft tissue. It also showed pathological fracture involving capitate with patchy areas of osteolysis (Fig. 3). Serial X-rays taken at 4 weeks, 8 weeks, 12 weeks, 18 weeks, and 6 months revealed that there was progressive concentric reduction of metacarpal shaft, first involving third then fourth metacarpal giving appearance of a sucked candy (Fig. 4). At this point in time, we kept in mind the differential diagnosis of primary bone tumor like aggressive giant cell tumor or aneurysmal bone cyst or metastasis from any other primary tumor. Positron emission tomography scan was done to rule out any tumor, however, no evidence of
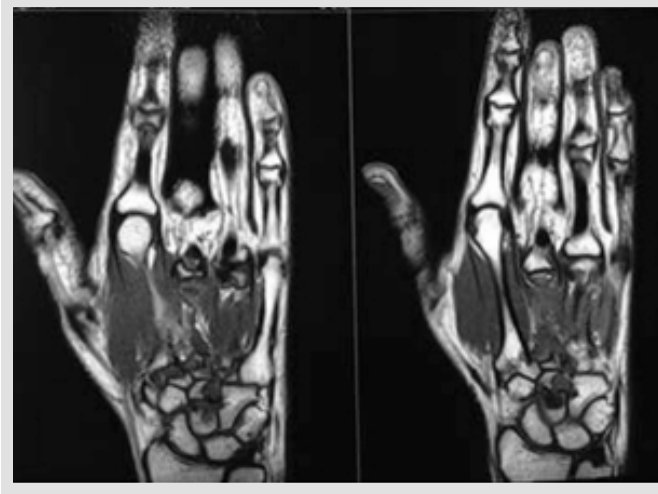

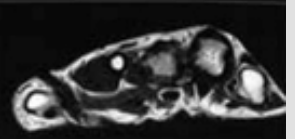

Figure 3: Magnetic resonance imaging images of the hand.
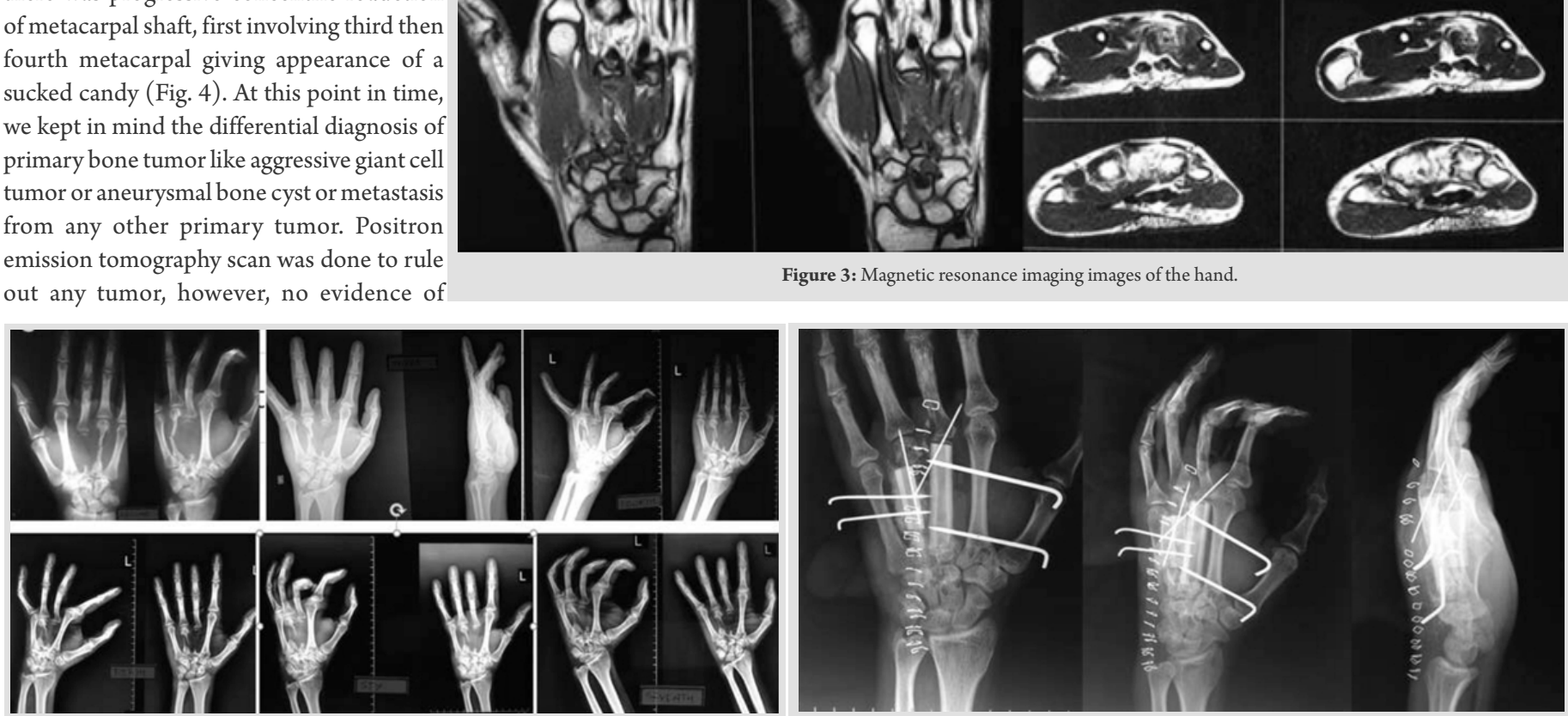

Figure 4: Serial radiographs showing progressive osteolysis.

Figure 5: Immediate post-operative radiograph

tumor was found. Parathyroid hormone levels were within normal limit. Biopsy suggested myxoid areas, proliferating vessels interposed with skeletal muscle fragments with no evidence of malignancy. Based on the clinical- bone graft substitute was used to fill cavity and some graft was put alongside of fibular graft. Postoperatively, hand and wrist were immobilized with plaster slab in functional position. The patient was started on $1000 \mathrm{mg}$ elemental

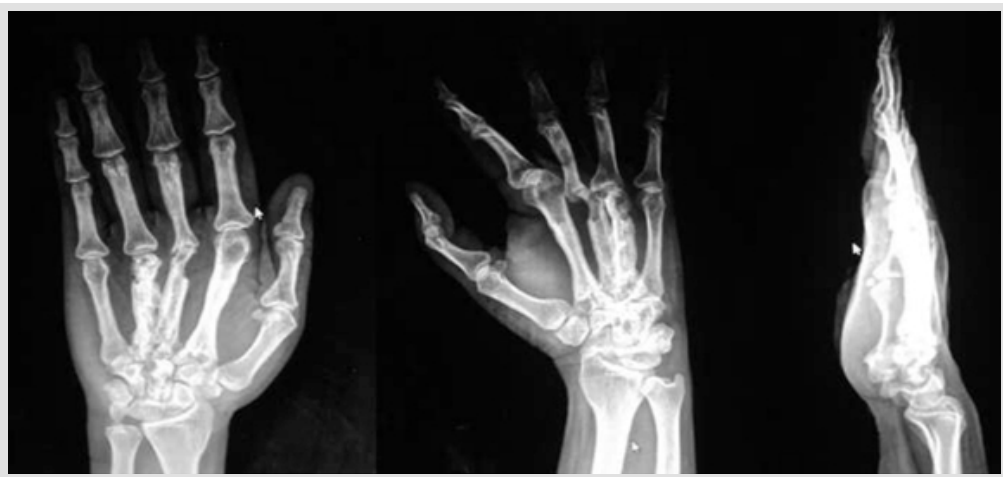

Figure 6: Radiograph at 2-year follow-up showing well incorporated of the fibular graft.

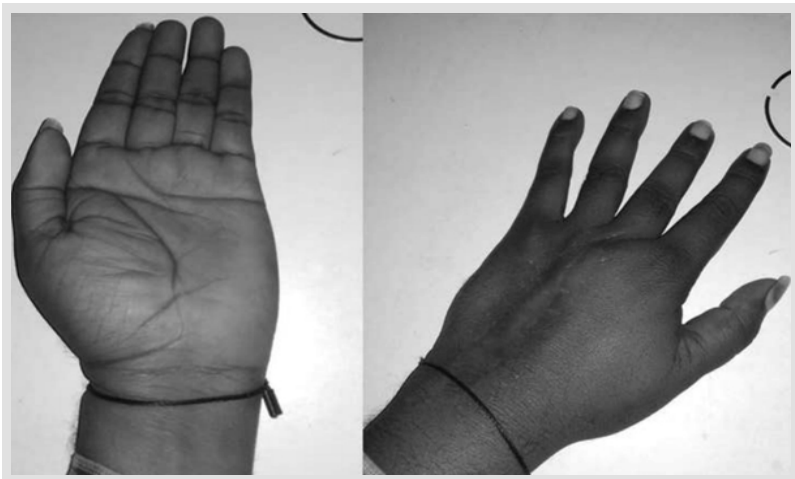

Figure 7: Clinical picture at 2-year follow-up. 
calcium in the form of calcium carbonate tablets. Vitamin D, 60,000 IU, once weekly for 12 weeks was also administered. Bisphosphonates were given in the form alendronate $70 \mathrm{mg}$ once weekly for 8 weeks. K-wires were removed at end of 8 weeks and gradual physiotherapy was started to improve hand function. At the end of 2 years, graft was completely incorporated (Fig. 6) and the patient gained functional recovery (Fig. 7) with active flexion at metacarpophalangeal joints of the $3 \mathrm{rd}$ and 4 th finger from 0 to $70^{\circ}$ and normal range at proximal interphalangeal (PIP) joint $\left(0-100^{\circ}\right)$ and DIP $\left(0-80^{\circ}\right)$ joints. The patient has $60 \%$ grip strength in affected hand as compared to normal hand (using dynamometer) with no evidence of recurrence (additional file 1: Movie 1).

\section{Discussion}

Diagnosis of vanishing bone disease should be made after excluding other mimicking condition such as metabolic bone disease (osteolysis secondary to hyperparathyroidism), inflammatory arthritis, and osteomyelitis [3]. The radiographic signs are osteolytic lesion that mimics localized osteoporosis which often progress to concentric complete bone resorption producing sucked candy appearance. Treatment of vanishing bone disease is difficult as there is a paucity of literature and no specific guidelines are recommended. Some case reports have advised use of bisphosphonate [6] (zoledronic acid infusion) and radiotherapy [7]. In our case, we have given intravenous zoledronic acid infusion along with calcium with Vitamin D supplements.
Recently, the use of interferons (alpha-2 b) and anti-platelet-derived growth factor monoclonal antibodies have been described in the literature [8]. Surgical resection of the lesion and reconstruction by bone graft or prosthesis can be done, however, prognosis is unpredictable.

\section{Conclusion}

Etiopathogenesis of vanishing bone disease is not fully understood and further research is needed for forming uniform and clear treatment guidelines. Vanishing bone disease affecting the metacarpal is very rarely reported in the literature and can be treated with non-vascularized fibular graft along with zoledronic acid, calcium, and Vitamin D supplementation.

\section{Clinical Message}

Diagnosis of vanishing bone diseases should be based on radiological, clinical, and histopathological findings. Early suspicion and surgical treatment in the form of strut bone graft (non-vascularized fibula graft) and ossifying therapy in the form of calcium and Vitamin D supplementation along with bisphosphonate therapy can achieve a favorable outcome in vanishing bone disease.

\section{References}

1. Gorham LW, Stout AP. Massive osteolysis (acute spontaneous absorption of bone, phantom bone, disappearing bone); its relation to hemangiomatosis. J Bone Joint Surg Am 1955;37:985-1004.

2. Nikolaou VS, Chytas D, Korres D, Efstathopoulos N. Vanishing bone disease (Gorham-stout syndrome): A review of a rare entity. World J Orthop 2014;5:694-8.

3. Möller G, Priemel M, Amling M, Werner M, Kuhlmey AS, Delling G. The Gorham-stout syndrome (Gorham's massive osteolysis). A report of six cases with histopathological findings. J Bone Joint Surg Br 1999;81:501-6.

4. Kiran DN, Anupama A. Vanishing bone disease: A review. J Oral Maxillofac Surg 2011;69:199-203.
5. Patel DV. Gorham's disease or massive osteolysis. Clin Med Res 2005;3:65-74.

6. Hammer F, Kenn W, Wesselmann U, Hofbauer LC, Delling G, Allolio B, et al. Gorham-Stout disease-stabilization during bisphosphonate treatment. J Bone Miner Res 2005;20:350-3.

7. Dunbar SF, Rosenberg A, Mankin H, Rosenthal D, Suit HD. Gorham's massive osteolysis: The role of radiation therapy and a review of the literature. Int J Radiat Oncol Biol Phys 1993;26:491-7.

8. Hagendoorn J, Yock TI, Rinkes IH, Padera TP, Ebb DH. Novel molecular pathways in Gorham disease: Implications for treatment. Pediatr Blood Cancer 2014;61:401-6.
Conflict of Interest: Nil Source of Support: Nil

Consent: The authors confirm that informed consent was obtained from the patient for publication of this case report

\section{How to Cite this Article}

Kamble P, Sathe A, Mohanty S, Rathod T. A Rare Case of Vanishing Bone Disease of Metacarpals. Journal of Orthopaedic Case Reports 2021 January;11(1): 101-103. 